\title{
Mobile Identity Management
}

\author{
Jaap-Henk Hoepman \\ TNO, Groningen, The Netherlands \\ Radboud University Nijmegen, The Netherlands \\ jhh@cs.ru.nl
}

\begin{abstract}
Identity management consists of the processes and all underlying technologies for the creation, management, and usage of digital identities. Business rely on identity management systems to simplify the management of access rights to their systems and services for both their employees and their customers. Users may benefit from identity management to simplify logging in to websites and computer systems (single sign-on), as well as streamlining management of their personal information and preferences (user centricity).

Current systems for identity management only partially achieve these goals, and still suffer from several security, privacy and usability issues. We will discuss how personal mobile devices (like mobile phones and PDA's) can be used to overcome this 'identity crisis', to increase the security, privacy and usability of identity management systems.
\end{abstract}

Short Biography. Jaap-Henk Hoepman is senior scientist computer security, privacy and identity management at TNO (the Dutch Organisation for Applied Scientific Research) and associate professor at the Radboud University Nijmegen, the Netherlands.

His research into information security and cryptographic protocols is inspired by practical problems. He focuses on the design of secure and privacy friendly protocols for the Internet of Things. Apart from that he studies privacy and identity management. He speaks on these topics at national and international congresses and publishes papers in (inter)national journals. He also appears in the media as security expert, and writes about his research in the popular press. He is actively involved in the public debate concerning security and privacy in our society. 